\title{
Research on UAV Decision Based on Weighted Distance Functions
}

\author{
Ruifeng Zhang"1, Shengkai Zhao", Shuqi Chen², Xinyu Lu ${ }^{3}$, Chenxi Du${ }^{1}$, * \\ ${ }^{1}$ School of Mathematics and Science, Qufu Normal University, Jining, Shandong, 273165, China \\ ${ }^{2}$ School of Statistics, Qufu Normal University, Jining, Shandong, 273165, China \\ ${ }^{3}$ School of Management, Qufu Normal University, Rizhao, Shandong, 273165, China \\ *Corresponding Author
}

\begin{abstract}
This paper mainly studies the optimal quantity, distribution model. This paper defined the working mechanism of cooperation between SSA UAV and radio relay UAV. We according to the distance relationship between different latitude and longitude on the earth, we use discretization to process a large number of data points which are used as discrete ignition points. The shortest weighted distance between EOC and each ignition point is obtained by Matlab software simulation. Finally, we given the optimal number and combination of UAV and radio relay UAV are 21 SSA UAV and 734 radio relay $U A V$.
\end{abstract}

Keywords: weighted distance function, UAV, simulation

\section{Introduction}

Firefighters use SSA drones to carry high-definition and thermal imaging cameras as well as telemetry sensors to monitor and report data from wearable devices for frontline personnel. Enable EOC to guide active crew to achieve the best results and maximum safety.

The Arcgic software is used to depict the Australian topographic map, and the Excel software is used to quantify the data. The relationship between latitude and longitude is obtained according to the knowledge of geography, the working mechanism of cooperation SSA UAV and radio relay UAV is clarified, a large number of data points are processed by discretization, the straight line is segmented with $5 \mathrm{~km}$ as the benchmark, and all endpoints are used as the discrete ignition points we need. For each, the new function which is the weighted distance function are defined; The frequency and size of fire events are considered as the parameters of the model, and the multi-objective decision problem is transformed into a single-objective decision problem by the deformation of the mathematical model. The shortest weighted distance between EOC and each ignition point is obtained by Matlab software simulation.

\section{Establishment of Model}

\subsection{Models that do not consider the frequency and magnitude of fire events as parameters}

According to the title, we lock the scope of the study in the fire area of Victoria, using Arcgic software, draw a topographic map of Australia, and get the following pictures:

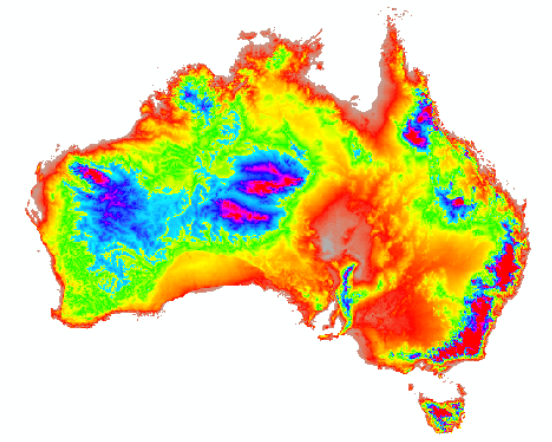

Figure 1: Distribution of ignition area in Australia [1] 
Based on the picture, we model with the help of scale and latitude and longitude.

First, the working mechanism of cooperation between SSA UAV and radio relay UAV is clarified.

SSA UAVs help monitor changing situations, according to the title description, so that the Emergency Operations Center (EOC) can best guide active aircrews to achieve the best results and maximum security; The hovering UAV with repeater is used to greatly expand the range of frontline low-power radios. Therefore, we can draw the following conclusions:

(1) SSA UAVs collect radio signals to collect ground firefighters' information and quickly collect ground personnel's information to EOC feedback;

(2) Since the range of transmission signals of SSA UAV is limited and affected by distance and terrain, the farther the distance is, the weaker the signal is, so it is necessary to use relay UAV to re-broadcast the signal cell phone and transmit the signal to the EOC as far as possible under the premise of ensuring the signal intensity;

(3) In order to ensure the effectiveness of signal transmission, a particular SSA UAV should fly along a fixed path on the premise of ensuring maximum flight capacity. And this path should fall as completely as possible within the range that the radio relay UAV can collect. If it is in a flat rural area, the trajectory of the SSA UAV should fall as completely as possible into the circle with the radio relay UAV corresponding to the point on the ground as the center and the radius of 20 $\mathrm{km}$;

Secondly, seriously consider the EOC location problem.

The original intention of the EOC is to grasp the fire situation in the shortest time and to give orders to the front line as soon as possible, so we must ask the EOC to collect all the information in the shortest time. For this purpose, considering that different terrain will interfere with the propagation and collection of signals, the shortest weighted distance between EOC and each ignition point must be considered. We define it as follows:

Based on what we have learned, we know that the traditional Euclidean distance is defined as follows:

$$
d(P, Q)=\sqrt{\left|x_{P}-x_{Q}\right|^{2}+\left|y_{P}-y_{Q}\right|^{2}}
$$

Where, the point coordinate $\mathrm{P}$ is $\left(x_{P}, y_{P}\right)$, the point coordinate $\mathrm{Q}$ is $Q\left(x_{Q}, y_{Q}\right)$.

Because the coordinates given in the title are longitude and latitude, and for the data points given in the figure above, the field distance corresponding to a longitude is not uniform with the field distance corresponding to a latitude. According to the knowledge of geography, we know that on the longitude line, the field distance is about $111 \mathrm{~km}$ for every 1 degree difference in latitude, and on the latitude line, the longitude is 1 degree difference, and the actual distance is $111 \cos s_{t}$ het $a \mathrm{~km}$.

Let $P\left(\xi_{P}, \eta_{P}\right)$ express the longitude and latitude of point $\mathrm{P}$ and $Q\left(\xi_{Q}, \eta_{Q}\right)$ express the longitude and latitude of the expression point $\mathrm{Q}$, then we can get the following relation:

$$
d(P, Q)=\left[\left(111 \cos 36^{\circ}\right)\left|\xi_{P}-\xi_{Q}\right|^{2}+111\left|\eta_{P}-\eta_{Q}\right|^{2}\right]^{\frac{1}{2}}
$$

In this process, we find that because of the relatively large number of data points collected, and some data points have been almost connected, which brings great inconvenience to our problem solving. We decided to discretize it. According to the ability of hand-held radio transmission and the terrain in which the approximate straight line is located are basically mountainous. We use 5 kilometers as a benchmark to segment the straight line. All endpoints are the discrete ignition points we need.

Because the range of repeater and SSA receiving and sending signals is restricted by topography and settlement form, we need to distinguish whether the target point is urban or rural and whether it is a mountain when we consider the problem. Therefore, we need to define a new class of functions, which we call "weighted distance functions ". The goal is to solve the most suitable EOC position so that the sum of" weighted distance functions "EOC reach each target point can be 
minimized.

Suppose that the index set of all points is obtained according to the data processing I, For each $\mathrm{i} \in \mathrm{I}$, the "weighted distance function" is defined as follows:

$$
\rho\left(E, \mathrm{~S}_{\mathrm{i}}\right)=\left\{\begin{array}{l}
2 d\left(E, S_{i}\right), S_{i} \text { is a country } \\
5 d\left(E, S_{i}\right), S_{i} \text { is a city and } \mathrm{d}\left(E, S_{i}\right) \leq 30 \mathrm{~km} \\
90+2 d\left(E, S_{i}\right), S_{i} \text { is a city and } d\left(E, S_{i}\right)>30 \mathrm{~km}
\end{array} .\right.
$$

Therefore, using the obtained data, the optimization model is as follows:

$$
\sum_{i \in I} \rho\left(E, \mathrm{~S}_{\mathrm{i}}\right)
$$

Thirdly, the optimal number and combination of SSA UAV and radio relay UAV are solved.

The number of SSA drones is relatively easy to find, and part of the number depends on the number of segments in the approximate line described above, just divided by 6 , but considering that the charging time is not much different from the maximum flight time, So we think about simplification, two SSA drones alternate every $30 \mathrm{~km}$, so we should divide them by 3 . The other part is a few scattered points, which need to calculate the distance between adjacent two points. If the interval is less than 30 kilometers, the two points share two SSA drones; otherwise, each point uses two SSA drones.

On the division of cities and rural areas, this paper according to the map of prefecture-level cities as cities, the rest of the areas collectively referred to as rural areas. According to the previous hypothesis three, the urban area is a disk area, which is the center of the longitude and latitude, with a radius of $30 \mathrm{~km}$. In order to facilitate the subsequent test of a large number of data models, the latitude and longitude of each city are kept integer, and the fire point is approximated by rounding method.

For the solution of the number of radio relay UAVs, we selected three groups of data for the fire in Victoria, Australia, 2019.10.11-2019.10.15, 2019.11-2019.11.15, 2019.12.11-12.15. The specific solution process will be presented in section 3 .

\subsection{Model considering the frequency and magnitude of fire events as parameters}

We rely on the corresponding data in (1) to obtain the reasonable location of the EOC at a certain time or in a short time and the optimal number and combination SSA UAV and radio repeater UAV under the corresponding conditions. In practice, the frequency and size of fire events are also changing over time. Therefore, when we consider, we should also make appropriate adjustments to the above model.

Since EOC can be moved, but for practical purposes, EOC need to be in a fixed position for a certain period of time, such as a day. Therefore, the frequency and size of fire events are particularly critical. The fire size of different locations can be replaced by the relevant indicators in the data table, and the fire frequency can be measured by the number of fires occurring within five days. We also need to maximize the degree of EOC perception of fire within its range for EOC to be as close as possible to the ignition point. The frequency of fire depicts the number of fires in a short period of time, and it is impossible to describe the situation of fires in a certain area in a period of time by virtue of this index alone. Therefore, we consider the product of fire frequency and related data index describing fire size, and add their product to the coefficient of Euclidean distance $\mathrm{E}$ each point in the model that did not consider fire frequency and size as parameters. For the safety of the EOC. So the definition of the weighted distance function is changed to $\mathrm{S}_{\mathrm{i}}, d\left(E, \mathrm{~S}_{\mathrm{i}}\right)$

$$
\rho\left(E, \mathrm{~S}_{\mathrm{i}}\right)=\left\{\begin{array}{l}
\left(2+f_{i} b_{i}\right) d\left(E, S_{i}\right), S_{i} \text { is a country } \\
\left(5+f_{i} b_{i}\right) d\left(E, S_{i}\right), S_{i} \text { is a city and } \mathrm{d}\left(E, S_{i}\right) \leq 30 \mathrm{~km} \\
90+\left(2+f_{i} b_{i}\right) d\left(E, S_{i}\right), S_{i} \text { is a city and } d\left(E, S_{i}\right)>30 \mathrm{~km}
\end{array} .\right.
$$

Thus, the final model is as follows: 


$$
\sum_{i \in I} \rho\left(E, \mathrm{~S}_{\mathrm{i}}\right) .
$$

\section{Analysis and solution of Model}

A. EOC Location: According to the diagram, select a reasonable representative point in the Excel, and the selection steps of the point are as Fig2.

(1) The latitude range is -39 to -32 , the longitude range is $140-158$, the time is $2019.11 .11-2019.11 .15$;

(2) Select longitude and latitude two columns, and then do scattered plot;

(3) Data processing: The data of all discrete points in scatter plot can be used directly;

The treatment of connected points in scattered plot:

(1) Subtract $5 /\left(111^{*} \cos\right.$ latitude) from the highest value according to the longitude index, because the latitude change is not particularly large, so we take the average 149 of the maximum and the minimum value, that is, subtract $5 /(111 * \cos 149$ from the highest value in turn, and then select the data that exactly matches in the table. If there is no data that exactly meets the requirements, it is replaced by adjacent data. Remove all unselected data.

(2)For the above indicators with a uniform longitude value, then arranged from high to low. From the highest value, subtract $5 / 11$ in turn, and then select the data that exactly matches in the table. If there is no data that exactly meets the requirements, it is replaced by adjacent data. Remove all unselected data.

Next, what we need to do is to solve the minimization model established in 2.1 using November data and apply it to December and October respectively.

The final results are as follows:

We use the simulated annealing algorithm in $\mathrm{C}++$ to obtain the shortest distance from the longitude and latitude of EOC $(-36.8526,144.736)$ to the total distance of each ignition point by the October data.According to the data of November, the position of EOC is $(-33.2585,150.669)$, and the sum of distance from each ignition point is the shortest $142 \mathrm{~km}$.According to the data of December, the position of EOC is $(-33.036,150.9872)$, and the sum of distance from each ignition point is the shortest of $109.0495 \mathrm{~km}$. The resulting picture is shown Fig3.

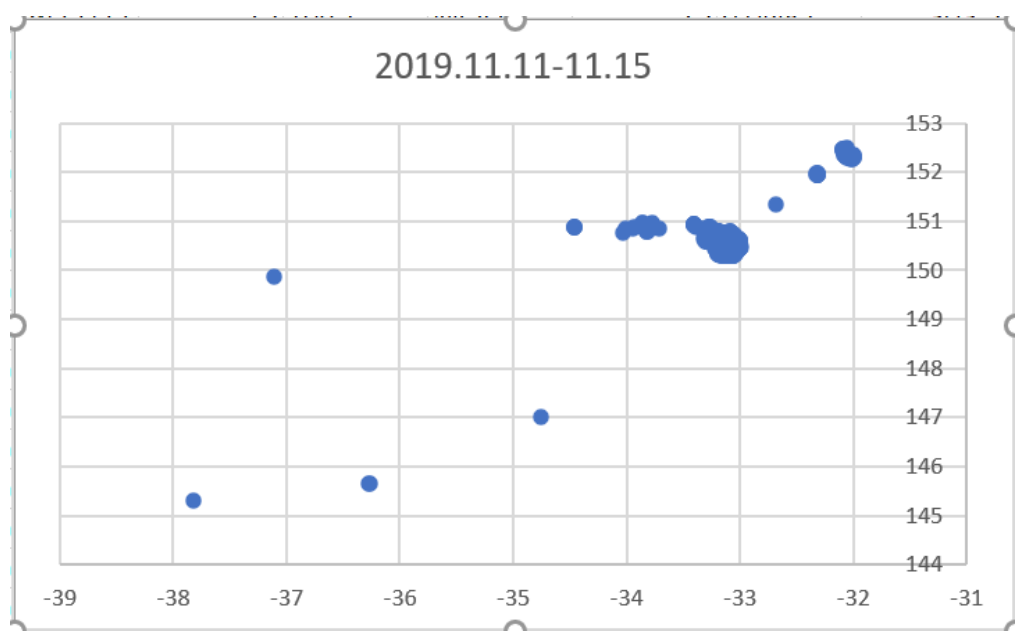

Figure 2: Location of ignition point, 2019.11-2019.11.15 [2] 


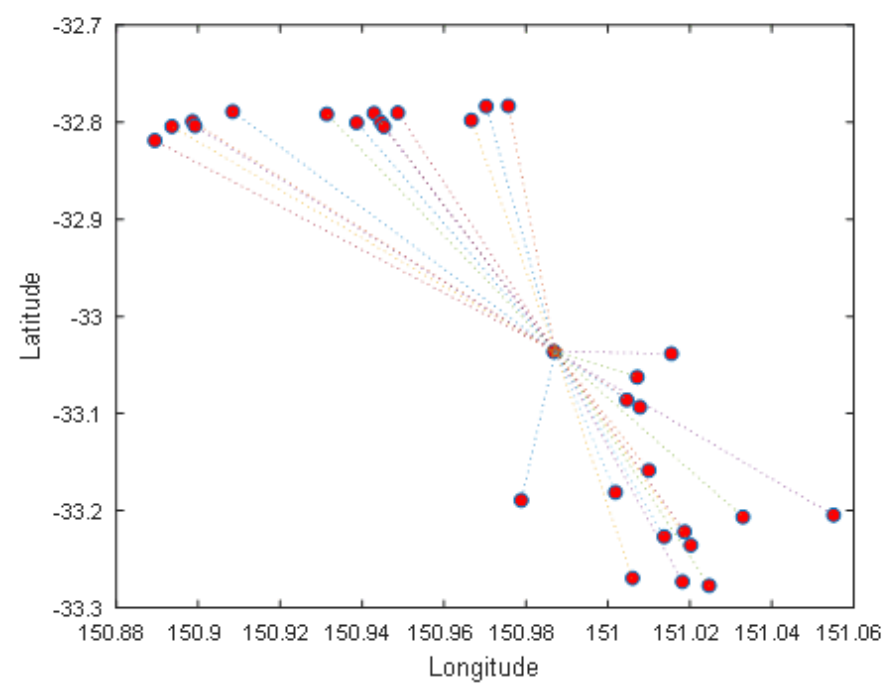

Figure 3: Visualization of EOC and each fire site in 2019 [2]

B. Determination of the optimal number and combination of SSA UAVs and radio repeater UAVs:

Taking 2019.11.11-2019.11.15 as an example, this paper describes how to calculate the number of SSA UAVs and the number of radio relay UAVs according to the distribution of ignition points within five days.

During this time, we finally screened out a total of 48 points together with two mountain tops, of which 7 discrete points, each of which required a SSA remaining 41 points required roughly 42 points Three $=14$ SSA drones. By this calculation, the total number of SSA drones is 21 .

In combination with the position of EOC determined in A, the linear distance between EOC and each discrete point is calculated. We assume that "the circle covered by two adjacent Repeaters is tangent", then we can know from the range of Repeaters is $20 \mathrm{~km}$. We just need to divide the linear distance by 40 to calculate the number of Repeaters. Using the programming algorithm, the minimum distance is 29328 and the number of Repeaters is 734 .

In summary, the optimal number and combination of UAVs and radio relay UAVs is 21 SSA UAVs and 734 Repeaters.

\section{References}

[1] Australian Meteorological Agency: http://www.bom.gov.au/.

[2] NASA: https://www.nasa.gov/.VIIRS 375 m Data from satellite instruments. 